\title{
AN ALGEBRAIC CHARACTERISATION OF CONVERGENCE IDEALS
}

\author{
BY JANE M. O. SPEAKMAN ${ }^{1}$
}

Communicated by M. Loève, August 25, 1966

We consider series whose terms are nonnegative real numbers. The terms of a series will be indexed by $Z$, the set of positive integers. Where there is no ambiguity we shall write $\sum_{A}$ for $\sum_{n \in A} a_{n}$ where $A$ is a subset of $Z$. The notation $A+B$ where $A$ and $B$ are sets will be used only when $A$ and $B$ are disjoint; it represents the union of $A$ and $B$. Even if $\sum a_{n}$ diverges there will be a large number of convergent subseries and the class $g$ of all the corresponding subsets of $Z$ forms an ideal (the convergence ideal). This means that (i) $A \in \mathfrak{g}$ and $B \subset A \Rightarrow B \in \mathfrak{g}$, (ii) $A$ and $B \in \mathfrak{g} \Rightarrow A \cup B \in \mathscr{g}$ and (iii) $\varnothing \in \mathfrak{g}$. Kakutani [1] has given a necessary and sufficient condition for two series to give rise to the same convergence ideal, but the problem of characterising convergence ideals appears to have been open until now, although some necessary and some sufficient conditions for an ideal to be a convergence ideal have been found by N. G. de Bruijn, P. Erdös, S. Kakutani and R. Rado (unpublished). This note describes a necessary and sufficient condition for an ideal to be a convergence ideal; this will be based on the new concept of a portabilityclass. A complete account of the work will be published elsewhere. ${ }^{2}$

Definition. A class $P$ of finite subsets of $Z$ is a portability-class if and only if it obeys rules $\left(1^{\circ}\right)-\left(6^{\circ}\right)$ below. The elements of the class will be called the portable sets.

$\left(1^{\circ}\right)$ All one-point sets are portable.

$\left(2^{\circ}\right)$ All subsets of portable sets are portable.

If $A$ and $B$ are portable sets and $D$ is a finite set disjoint from $A$ and $B$ such that $A+D$ is portable while $B+D$ is not, then

$\left(3^{\circ}\right)$ for all finite sets $E$ disjoint from $A$ and $B$ either $A+E$ is portable or $B+E$ is not, and

$\left(4^{\circ}\right)$ given any finite set $C$ there exists a finite set $F$ disjoint from $A \cup B \cup C$ such that $A+F$ is portable while $B+F$ is not.

Under $\left(1^{\circ}\right)-\left(4^{\circ}\right)$ we can define a relation on the portable sets by saying that $A$ is not heavier than $B$, written $A \leqq B$, if and only if, for

1 This work was done during the tenure of a Science Research Council Studentship and a research studentship from Girton College.

2 The paper is to appear in the Journal of the London Mathematical Society. 
all finite $D$ disjoint from $A$ and $B, B+D \in \odot$ implies that $A+D \in \odot$. This relation is a total quasi-ordering, i.e., it is reflexive and transitive, and for all $A$ and $B \in \mathcal{P}$ either $A \leqq B$ or $B \leqq A$ or both. When $A \leqq B \leqq A$ we write $A \cong B$. This is an equivalence relation on 8 .

$\left(5^{\circ}\right)$ If $A$ and $B$ are disjoint portable sets such that $A+B$ is not portable, then for any finite $E$ there exists a portable set $C$ disjoint from $A \cup E$ such that $A+C \notin \odot$ and $C \leqq B$.

$\left(6^{\circ}\right)$ If $A$ is portable and $A \neq \varnothing$, then there exists an integer $k$ and $k$ disjoint portable sets each not heavier than $A$ such that their union is not portable.

Rule $\left(6^{\circ}\right)$ is a condition of Archimedean type.

We now state our main result.

THEOREM. A necessary and sufficient condition that an ideal $g$ of subsets of $Z$ be the ideal of convergence sets for a series of nonnegative terms is that there should exist a portability-class $\odot$ such that the limits of increasing seguences of elements of $\odot$ generate 9.

Here, when we say "increasing sequence," we mean increasing in the sense of the ordinary set-theoretic partial ordering based on inclusion. If the limits of increasing sequences of elements of $P$ constitute the class $\mathscr{L}$, then the condition of the theorem means that $E \in \mathscr{g}$ iff $E$ is the union of finitely many $\mathcal{L}$-sets. Further it can be shown that $E \in \mathcal{I}$ iff $E$ differs from some element of $\mathcal{L}$ by a finite set.

In the proof of the necessity of the condition the most interesting case is when $\sum a_{n}$ has the properties that $a_{n} \leqq 1$ and, for all $\delta>0$, $\sum_{\left\{a_{n}<\delta\right\}}$ is divergent. In that case we can obtain a portability class $\beta$ of the required type by saying that a finite set $A \in \mathcal{P}$ iff $\sum_{A} \leqq 1$.

To establish sufficiency, given $\rho$ we construct a series $\sum a_{n}$ by taking $a_{n}$ to be 0 if $\{n\} \cong \varnothing$ and a function of the least $k$ which satisfies $\left(6^{\circ}\right)$ with $A=\{n\}$ otherwise.

I am very grateful to Professor D. G. Kendall for his advice and encouragement.

\section{REFERENCE}

1. S. Kakutani, Note on divergent series and integrals, Proc. Imp. Acad. Tokyo 20 (1944), 74-76.

Statistical Laboratory, University of Cambridge, England 